February 2000

Reply to A. Patrascioiu's and E. Seiler's comment on our paper

\title{
Percolation properties of the 2D Heisenberg model
}

\author{
B. Allés ${ }^{\mathrm{a}}$, J. J. Alonso ${ }^{\mathrm{b}}$, C. Criado ${ }^{\mathrm{b}}$, M. Pepe ${ }^{\mathrm{c}}$ \\ ${ }^{a}$ Dipartimento di Fisica, Università di Milano-Bicocca \\ and INFN Sezione di Milano, Milano, Italy \\ ${ }^{\mathrm{b}}$ Departamento de Física Aplicada I, Facultad de Ciencias, 29071 Málaga, Spain \\ ${ }^{\mathrm{c}}$ Institut für Theoretische Physik, ETH-Hönggerberg, CH-8093 Zürich, Switzerland
}

The most of the problems raised by the authors of the comment [ [] about Ref. [2] are based on claims which have not been written in [2], for instance almost all the introduction and the point (1) in [1] are based on such nonexistent claims.

Instead in Ref. [2] we avoid to make claims not based on well-founded results. For instance in the abstract we write "... This result indicates how the model can avoid a previously conjectured Kosterlitz-Thouless $[K T]$ phase transition..." and in the conclusive part we notice that "Our results exclude this massless phase for $T>0.5$ ". Therefore it seems to us that the opening sentence in the Comment [1] "In a recent letter Allés et al. claim to show that the two dimensional classical Heisenberg model does not have a massless phase." is strongly inadequate.

As for the points that appear in the Comment:

- (1) The purpose of the paper [2] is to fill a gap in the research about the critical properties of the Heisenberg model. This gap is the following one: in Ref. [3] a scenario was proposed where the 2D Heisenberg model should undergo a KT phase transition at a finite temperature. This scenario is based mainly on three hypotheses, the third one (which states the non-percolation of the $\mathcal{S}$-type or equatorial clusters) being left in [3] without a plausible justification. To back up that hypothesis a numerical 
test was cited in [3] but the details of the numerics (temperature, size of the lattice, etc.) and several data concerning the percolation properties of the system, were completely skipped. The only quoted result was (see beginning of section 4 in [3]) "We also tested numerically for $\epsilon=1 / 3, \ldots$ There is no indication of percolation...". On the contrary, such interesting results about the critical properties should be put forward with a thorough description of the hypotheses involved. Moreover, one would like to understand how was possible to use the small value of epsilon mentioned in Ref. [3], because that value implies a really tiny temperature $T$ and consequently it requires a huge lattice size. If "Everybody agrees that at $\beta=2.0$ the standard action model has a finite correlation length", see [1], also everybody would like to know details about the numerics and the computer used to simulate the model at such a small temperature.

- (2) There is a statement in [2] which is repeated several times: all results are valid for any versor $\vec{n}$ of the internal symmetry space $O(3)$. In particular, a percolating equatorial cluster is found for every $\vec{n}$. Under these conditions, we do not see how the percolation of the equatorial cluster may lead to a breaking of the $O(3)$ symmetry.

On the other hand, the fractal properties of a cluster are very sensitive to the choice of parameters. By varying $\epsilon$ around the value $\epsilon=1$ (for $T=$ $0.5)$, one can make the data for $\left\langle M_{\mathcal{S}}\right\rangle / L^{2}$ in Table 1 of [2] to change rather dramatically. It is important (even in the case of a high temperature regime, like $T=0.5$ ) to study this dependence. It is sensible to expect that the fractal properties of the cluster show up at the threshold of percolation. Again in [2] we do not claim that the cluster is a fractal, but just write "... [the equatorial clusters] present a high degree of roughness recalling a fractal structure". To state any firmer claim, a deep analysis of the errors and better statistics in Table 1 should be performed. All these problems are currently investigated.

- (3) It is true that not all flimsy clusters can avoid a KT transition. However this trivial truth proves nothing. Other kinds of lattices can hold versions of the $X Y$ model with no transition (see for instance [4]).

On the other hand, the statement "... there should be no doubt that on such a lattice [square holes of side length $L$ ] the $O(2)$ model has a KT phase transition for any finite $L "$ is surprising. In Ref. [5] it is shown that for any finite $L$ the KT transition is still present but it approaches $T=0$ as $L$ becomes larger. The idea of a fractal as the limit of some kind of cluster should not be forgotten.

- (4) We agree with one of the sentences of this point: "It would be in- 
teresting to verify this [the existence of a KT transition for $X Y$ models on a fractal lattice]". Yet we do not see the relevance of such an obvious claim.

We disagree however with the authors of [1] when they say "our argument does not depend on the existence of such a transition on that particular percolating cluster". Instead, after the conclusions of Ref. [2], we think that the non-rigorous proof proposed in [3] for the case when the equatorial cluster does percolate, heavily lies on whether or not such a transition is realized.

\section{References}

[1] A. Patrascioiu and E. Seiler, unpublished report hep-lat/9912014 (v1).

[2] B. Allés, J.J. Alonso, C. Criado and M. Pepe, Phys. Rev. Lett. 83 (1999) 3669 .

[3] A. Patrascioiu and E. Seiler, Nucl. Phys. B (Proc. Suppl.) 30 (1993) 184.

[4] Yu.E. Lozovik and L.M. Pomirchy, Solid State Comm. 89 (1994) 145.

[5] P. Minnhagen and H. Weber, Physica B152 (1988) 50. 